\title{
Different Clinical and Neuroimaging Characteristics in Early Stage Parkinson's Disease with Dementia and Dementia with Lewy Bodies
}

\author{
Mami Takemoto, Kota Sato, Noriko Hatanaka, Toru Yamashita, Yasuyuki Ohta, \\ Nozomi Hishikawa and Koji Abe* \\ Department of Neurology, Graduate School of Medicine, Dentistry and Pharmaceutical Sciences, Okayama \\ University, Kita-ku, Okayama, Japan
}

Accepted 19 January 2016

\begin{abstract}
Parkinson's disease with dementia (PDD) and dementia with Lewy bodies (DLB) both commonly exhibit brain Lewy body pathology and similar end-stage symptoms, but early symptoms differ. To clarify these differences, we compared the demographic characteristics, symptoms, cognitive and affective functioning, activities of daily life, and neuroimaging results between PDD $(n=52)$ and DLB $(n=46)$ patients. In measures of cognitive functioning, PDD patients had worse Hasegawa dementia scale-revised (HDS-R) scores (11.2 \pm 4.8$)$ and better frontal assessment battery (FAB) scores (11.3 \pm 4.1$)$ compared with DLB $(17.0 \pm 6.4, p=0.013$ and $8.6 \pm 4.7, p=0.039$, respectively). DLB patients performed worse than PDD patients in "orientation to place" tasks. In affective functions, DLB patients had worse GDS (7.6 \pm 3.4$)$ and ABS $(9.9 \pm 5.3)$ scores than PDD patients $\left(5.1 \pm 4.1\right.$ and $4.8 \pm 3.0$, respectively). ${ }^{99 \mathrm{~m}} \mathrm{Tc}-\mathrm{ECD}$ images showed greater CBF in the whole cingulate gyrus and a lower CBF in the precuneus area in DLB than in PDD. These results suggest that PDD patients' lower average scores for "repetition" (MMSE), "recent memory" (HDS-R), and "lexical fluency" (FAB) were related to lower CBF in the cingulate gyrus than in DLB. Furthermore, DLB patients' poorer average subscale scores of "orientation to place" (MMSE) and "similarities", "conflicting instructions", and "go-no go" (FAB) tasks may be related to the lower CBF in the precuneus area in DLB than PDD.
\end{abstract}

Keywords: Affective function, cognitive function, dementia with Lewy bodies, Parkinson's disease with dementia, ${ }^{99 \mathrm{~m}} \mathrm{Tc}-\mathrm{ECD}$ images

\section{INTRODUCTION}

Parkinson's disease (PD) is an age-related degenerative disorder characterized by a profound loss of dopamine producing neurons, with Lewy body inclusions, mainly in the substantia nigra and basal ganglia. PD patients develop motor symptoms such

\footnotetext{
*Correspondence to: Koji Abe, Department of Neurology, Graduate School of Medicine, Dentistry and Pharmaceutical Sciences, Okayama University, 2-5-1 Shikata-cho, Kita-ku, Okayama 700-8558, Japan. Tel.: +81 86235 7365; Fax: +81 86 235 7368; E-mail: takemoto.demekin@gmail.com.
}

as resting tremor, rigidity, bradykinesia, and postural instability as well as non-motor symptoms such as cognitive impairment, depression, apathy, and autonomic dysfunctions [1-3].

Parkinson's disease with dementia (PDD) is a late complication of PD due to cerebral cortical degeneration, commonly occurring after a disease duration of about 10 years, and with a cumulative prevalence of 75-90\% [4]. Dementia with Lewy bodies (DLB) is also a common type of neurodegenerative dementia, along with Alzheimer's disease [5]. DLB and PD both commonly show Lewy body pathology, which 
is more severe in the neocortex in DLB than in PD. Clinically, PDD and DLB are distinguished based on whether Parkinsonism precedes dementia by more than a year.

Although patients with PDD and DLB develop similar symptoms in the advanced stages, symptoms in the early stages differ. A previous report showed that cognitive dysfunction is greater in DLB than in PDD and relates to frontal and medial temporaldysfunction [6]. Patients with DLB have decreased cerebral blood flow (CBF) in the occipital lobe, percutaneous gyrus, and posterior cingulate cortex $[7,8]$, whereas PDD presents with decreased CBF in the posterior cingulate cortex, precunei, and bilateral parietotemporal association cortices [9].

Recent studies have demonstrated that the antidementia drug, donepezil, improves cognition and executive function in PDD [10] and DLB [11], being more beneficial in the latter [12]. However, these data were not comprehensively obtained from the physical, cognitive, affective, and brain symptoms, age of onset, period to diagnosis of dementia, age of dementia onset, Yahr grade, cognitive and affective functioning, and activities of daily living (ADL) scores to investigate the differences in the early stages of PDD and DLB.

\section{MATERIALS AND METHODS}

\section{Participants}

We evaluated 52 PDD patients (30 men and 22 women; age, $73.8 \pm 8.4$ years old) and 46 agematched DLB patients (19 men and 27 women; age, $72.2 \pm 7.1$ years) who visited Okayama University Hospital, Okayama, Japan, and its affiliated hospitals between June 2010 and March 2014. We diagnosed PDD and DLB according to the clinical diagnostic criteria for PDD [12] and the clinical criteria of the Consortium on DLB [13]. The demographic characteristics of PDD and DLB patients were compared for their ages of onset, periods to diagnosis of dementia, ages at diagnosis of dementia, Yahr grade, Parkinsonian symptoms, autonomic disturbance, and initial dementia symptom(s). The period to diagnosis of dementia was defined as being from the time of PD diagnosis, or in DLB from the onset of preclinical suggestive symptoms (i.e., delusions, hallucinations, floating sensations, tremor, rigidity, akinesia, or postural instability), to the development of dementia as assessed by a Mini-Mental State Examination (MMSE) score of $<23$. This study was approved by the Ethical Committee of Graduate School of Medicine, Dentistry and Pharmaceutical Science, Okayama University (approval \#777).

\section{Cognitive and affective functioning}

Cognitive functioning was assessed using the MMSE, Hasegawa dementia score-revised (HDS-R), frontal assessment battery (FAB), and Montreal cognitive assessment (MoCA). Affective functioning was assessed using the geriatric depression scale (GDS), apathy scale (AS), and Abe's behavioral and physiological symptoms of dementia score (ABS). Activities of daily living (ADL) were assessed using the Alzheimer's disease Cooperative StudyActivities of Daily Living scale (ADCS-ADL).

\section{Neuroimaging}

Brain MRI scans were obtained from 46 PDD and 40 DLB patients to assess white matter changes using the Fazekas scale [14]. Periventricular hyperintensity $(\mathrm{PVH})$ and deep white matter hyperintensity (DWMH) lesions were graded as 0-III for PVH and 0-3 for DWMH. Myocardial ${ }^{123}$ I-metaiodobenzylguanidine (MIBG) images for heart-to-mediastinum $(\mathrm{H} / \mathrm{M})$ ratio were also obtained from $10 \mathrm{PDD}$ and $12 \mathrm{DLB}$ patients with exclusion criteria of cardiac failure, ischemic heart disease, poorly controlled diabetes mellitus (HbA1c>7.0\%), insulin therapy, and severe kidney dysfunction.

To compare $\mathrm{CBF},{ }^{99 \mathrm{~m}} \mathrm{Tc}$-ethylcysteinate dimer (ECD) SPECT scintigraphs [15] were obtained from 15 PDD and 19 DLB patients. The SPECT images were anatomically standardized for using SPM5 [16]. In this method, an averaged SPECT image of DLB and PDD were created from these anatomically standardized images and scaling voxel counts by a global mean. We then evaluated a subtraction image of averaged PDD and DLB SPECT images.

\section{Statistical analysis}

Statistical analyses were performed using SPSS (22.0.0.0; IBM, Armonk, NY, USA). The MannWhitney U test was used to compare cognitive, affective, and ADL functioning, along with neuroimaging results, between the PDD and DLB groups. Chisquare tests were used to compare Parkinsonian symptoms (tremor, autonomic disturbance, and floating sensation). 
Table 1

Demographic characteristics of PDD and DLB patients

\begin{tabular}{|c|c|c|c|c|}
\hline & & PDD & DLB & $p$ value \\
\hline $\mathrm{n}$ (male) & & $52(57.6 \%)$ & $46(41.3 \%)$ & \\
\hline Age of onset (y) & & $73.8 \pm 8.4$ & $72.2 \pm 7.1$ & 0.609 \\
\hline Period to diagnosis (y) & & $6.2 \pm 5.1$ & $0.6 \pm 0.4$ & $<0.001^{* *}$ \\
\hline Age of dementia (y) & & $80.0 \pm 13.5$ & $72.8 \pm 7.5$ & 0.461 \\
\hline Yahr grade & & $2.8 \pm 0.8$ & $2.4 \pm 0.8$ & 0.069 \\
\hline Yahr $1(\%)$ & & 5.0 & 13.5 & \\
\hline Yahr $2(\%)$ & & 30.6 & 32.4 & \\
\hline Yahr $3(\%)$ & & 40.8 & 40.5 & \\
\hline Yahr $4(\%)$ & & 18.4 & 5.4 & \\
\hline UPDRS (partIII) & & $46.3 \pm 10.9$ & $37.9 \pm 13.1$ & $<0.001^{* *}$ \\
\hline Parkinsonism (\%) & at disease onset & at developing dementia & & \\
\hline Tremor & 88.0 & 88.0 & 19.0 & $<0.001^{* *}$ \\
\hline Rigidity & 75.0 & 85.0 & 74.0 & 0.289 \\
\hline Akinesia & 6.0 & 15.0 & 19.0 & 0.783 \\
\hline Postural instability & 0.0 & 58.0 & 33.0 & $0.022^{*}$ \\
\hline \multicolumn{5}{|l|}{ Autonomic disturbance $(\%)$} \\
\hline Orthostatic hypotension & & 7.0 & 78.0 & $<0.001^{* *}$ \\
\hline Urinary disturbance & & 32.0 & 17.0 & 0.379 \\
\hline Constipation & & 71.0 & 65.0 & 0.863 \\
\hline \multicolumn{5}{|c|}{ Initial symptom of dementia (\%) } \\
\hline Delusion & & 13.0 & 78.0 & $<0.001^{* *}$ \\
\hline Hallucination & & 6.0 & 17.0 & 0.134 \\
\hline Floating sensation & & 2.0 & 65.0 & $<0.001^{* *}$ \\
\hline
\end{tabular}

Data represent mean \pm SD.

\section{RESULTS}

The demographic characteristics, clinical course (ages of onset, periods to diagnosis of dementia, ages of dementia onset), Yahr grade, Parkinsonism, autonomic disturbance, and initial symptoms of dementia are presented in Table 1. There were no significant differences in age of onset, age of dementia and Yahr grade between PDD and DLB patients.

PDD patients more commonly experienced tremor $(88.0 \%)$ than DLB patients $(19.0 \%, p<0.001)$. In contrast, DLB patients more commonly had orthostatic hypotension $(\mathrm{OH}, 78.0 \%)$, as a measure of autonomic disturbance, than PDD patients $(7.0 \%$, $p<0.001)$. The DLB group also had higher rates of delusion (78.0\%), hallucination (17.0\%), and floating sensation (65.0\%) symptoms than the PDD group $(13.0 \%, 6.0 \%$, and $2.0 \%$, respectively).

As shown in Table 2, PDD patients had similar MMSE but worse HDS-R $(11.2, p=0.013)$ scores than DLB patients (17.0). In contrast, the average FAB score was significantly higher in PDD $(11.3 \pm 4.1, p=0.039)$ than DLB patients $(8.6 \pm 4.7)$. As measures of affective functioning, DLB patients had worse GDS $(7.6 \pm 3.4, p=0.023)$ and ABS $(9.9 \pm 5.3, p<0.001)$ scores than in PDD patients
(5.1 \pm 4.1 and $4.8 \pm 3.0$, respectively). The PDD group had poorer ADL results $(13.5 \pm 6.8, p=0.041)$ than the DLB group (16.8 \pm 5.6). MRI-based Fazekas scale and MIBG scintigraphy were not significantly different between the two groups (Table 2, bottom).

Table 3 shows the MMSE, HDS-R, and FAB subscale scores in PDD and DLB patients. On the MMSE and HDS-R subscales, DLB patients had poorer scores only in "orientation to place" $(3.3 \pm 1.5$, $p=0.016$ and $1.6 \pm 0.7, p=0.011)$ when compared with PDD patients $(4.3 \pm 1.1$ and $2.0 \pm 0.0)$. However, DLB patients had worse scores on three FAB subscales: "similarities", "conflicting instructions", and "go-no go" $(1.9 \pm 1.2,0.9 \pm 1.0$, and $0.1 \pm 0.2$, $p=0.039, p=0.016$, and $p=0.004$ ) than PDD patients $(2.1 \pm 1.0,1.8 \pm 1.3$, and $1.1 \pm 1.4)$.

Figure 1 (left) shows ${ }^{99 \mathrm{~m}}$ Tc-ECD SPECT images of the mean CBF values in PDD and DLB patients. The comparison between the two images revealed a slight decrease in the frontal lobe in PDD, but similar $\mathrm{CBF}$ in both posterior lobes. However, the subtraction image of mean PDD - mean DLB (Figure, right) shows higher $\mathrm{CBF}$ in the whole cingulate gyrus in DLB than in the PDD group (arrowheads). In contrast, there is less CBF in the precuneus area in DLB (arrows) than PDD. 
Table 2

Cognitive, affective, and ADL functions with MRI-based Fazekas scale and MIBG scintigraphy

\begin{tabular}{lrrc}
\hline & \multicolumn{1}{c}{ PDD } & \multicolumn{1}{c}{ DLB } & $p$ value \\
\hline MMSE & $19.8 \pm 3.6$ & $19.1 \pm 6.5$ & 0.954 \\
HDS-R & $11.2 \pm 4.8$ & $17.0 \pm 6.4$ & $0.013^{*}$ \\
FAB & $11.3 \pm 4.1$ & $8.6 \pm 4.7$ & $0.039^{*}$ \\
MoCA & $15.5 \pm 4.3$ & $18.3 \pm 4.0$ & 0.581 \\
GDS & $5.1 \pm 4.1$ & $7.6 \pm 3.4$ & $0.023^{*}$ \\
Apathy & $14.4 \pm 8.9$ & $20.1 \pm 9.9$ & 0.080 \\
ABS & $4.8 \pm 3.0$ & $9.9 \pm 5.3$ & $<0.001^{* *}$ \\
ADL & $13.5 \pm 6.8$ & $16.8 \pm 5.6$ & $0.041^{*}$ \\
Fazekas PVH & $1.2 \pm 1.0$ & $1.3 \pm 1.0$ & 0.571 \\
Fazekas DWMH & $1.5 \pm 1.0$ & $1.6 \pm 1.0$ & 0.497 \\
MIBG, early & $1.8 \pm 0.4$ & $1.6 \pm 0.5$ & 0.892 \\
MIBG, delayed & $1.6 \pm 0.5$ & $1.3 \pm 0.1$ & 0.311 \\
MIBG, washout rate $(\%)$ & $42.3 \pm 13.3$ & $47.3 \pm 9.4$ & 0.376 \\
\hline
\end{tabular}

Data represent mean $\pm \mathrm{SD}$.

Table 3

Subscales of MMSE, HDS-R, and FAB in PDD and DLB patients

\begin{tabular}{llccc}
\hline & & & DLB & $p$ value \\
\hline MMSE & orientation to time & $3.5 \pm 1.3$ & $1.6 \pm 0.7$ & 0.211 \\
& orientation to place & $4.3 \pm 1.1$ & $3.3 \pm 1.5$ & $0.016^{*}$ \\
& registration & $2.8 \pm 0.6$ & $2.9 \pm 0.6$ & 0.564 \\
& attention and calculations & $1.4 \pm 1.4$ & $1.4 \pm 1.5$ & 0.736 \\
& recent memory & $1.3 \pm 1.0$ & $1.4 \pm 1.0$ & 0.626 \\
& language & $1.9 \pm 0.4$ & $1.8 \pm 0.6$ & 0.333 \\
& repetition & $0.7 \pm 0.4$ & $0.8 \pm 0.8$ & 1.000 \\
HDS-R & drawing interlocking pentagons & $0.7 \pm 0.5$ & $0.5 \pm 0.5$ & 0.283 \\
& age & $0.9 \pm 0.3$ & $0.8 \pm 0.4$ & 0.774 \\
& orientation to time & $2.7 \pm 1.2$ & $2.0 \pm 1.5$ & 0.160 \\
& orientation to place & $2.0 \pm 0.0$ & $1.6 \pm 0.7$ & $0.011^{*}$ \\
& calculation & $1.0 \pm 0.7$ & $0.9 \pm 0.6$ & 0.505 \\
FAB & recent memory & $3.3 \pm 1.9$ & $3.5 \pm 1.9$ & 0.706 \\
& memory of 5 items & $3.4 \pm 1.5$ & $3.3 \pm 1.7$ & 0.974 \\
& frontal lobe function & $3.5 \pm 2.0$ & $2.2 \pm 2.2$ & 0.077 \\
& similarities & $2.1 \pm 1.0$ & $1.9 \pm 1.2$ & $0.039^{*}$ \\
& lexical fluency & $1.3 \pm 1.1$ & $1.5 \pm 1.2$ & 0.769 \\
& programming & $2.0 \pm 1.1$ & $1.6 \pm 1.2$ & 0.606 \\
& conflicting instruction & $1.8 \pm 1.3$ & $0.9 \pm 1.0$ & $0.016^{*}$ \\
& go-no go (inhibitory control) & $1.1 \pm 1.4$ & $0.1 \pm 0.2$ & $0.004^{* *}$ \\
& prehension behavior & $2.7 \pm 0.7$ & $2.7 \pm 0.8$ & 0.508 \\
\hline
\end{tabular}

Data represent mean $\pm \mathrm{SD}$.

\section{DISCUSSION}

As the clinical course for DLB patients' is very rapid (in our research, years from "from the first onset of a symptom that indicated possible DLB" to "diagnosed with dementia: MMSE $<23$ " was $0.6 \pm 0.4$ years), diagnoses in the early stage, and distinguishing from PDD, are very important for preparing for their terminal dementia stage.

We found that PDD patients had similar cognitive MMSE and MoCA scores, poorer HDS-R, and better FAB scores than DLB patients (Table 2). Of interest was that the subscale analyses showed a common decrease in "orientation to place" in both the MMSE and HDS-R scales (Table 3). Three items of the FAB subscale, "similarities", "conflicting instructions", and "go-no go", were better in PDD than in DLB (Table 3). These data are similar to a previous report showing that deficits in frontal and medial temporal-related cognitive functions are greater in DLB than in PDD patients [6].

Our DLB patients had poorer scores in all three affective factor items, particularly as assessed by the GDS and ABS (Table 2). Previous studies have shown that $80 \%$ of DLB patients have combined depressive and psychiatric symptoms [17], with only 20-50\% of PD patients showing depressive symptoms [18]. In the present study, we used ABS for evaluating the behavioral and psychological symptoms of dementia [19], and it revealed the strongest 
PDD mean
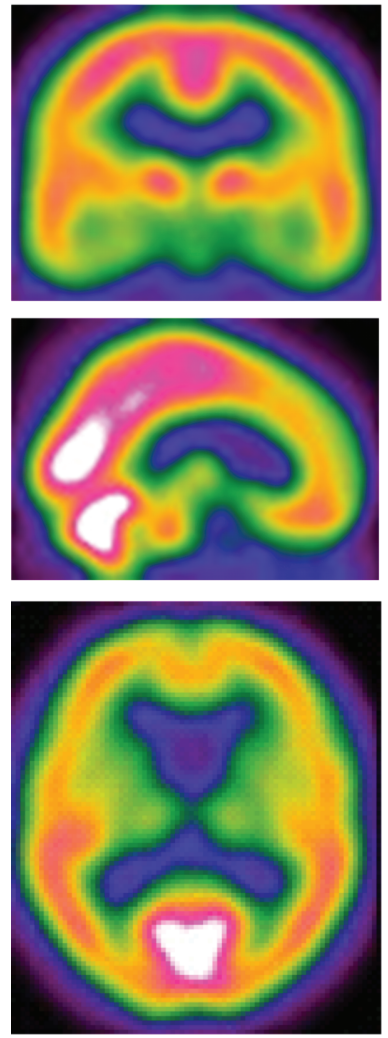

DLB mean
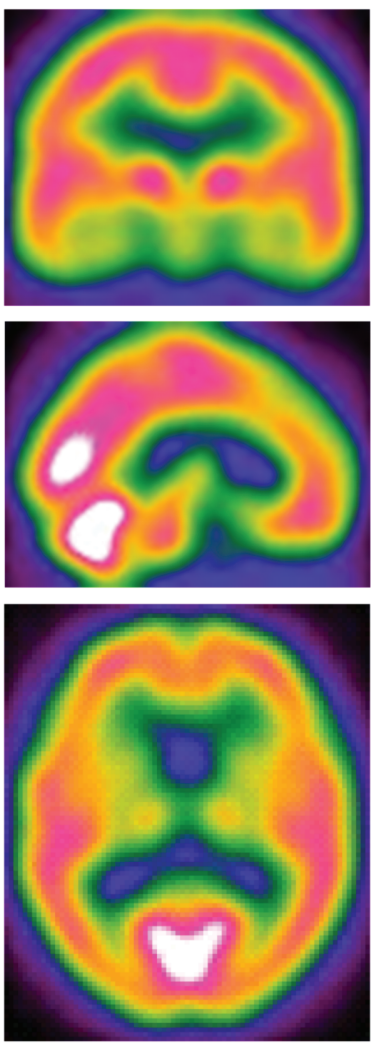
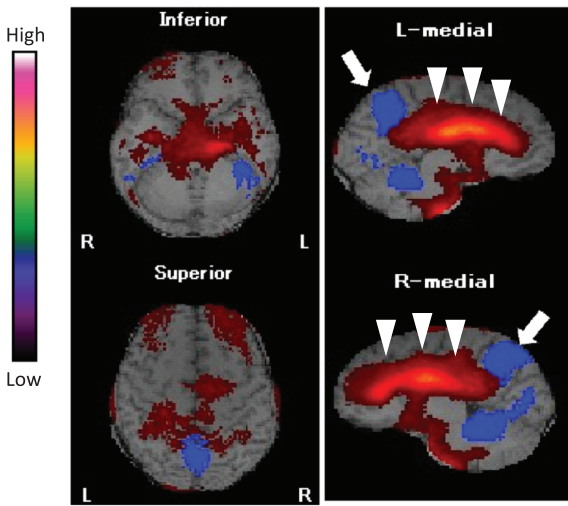

DLB $>$ PDD
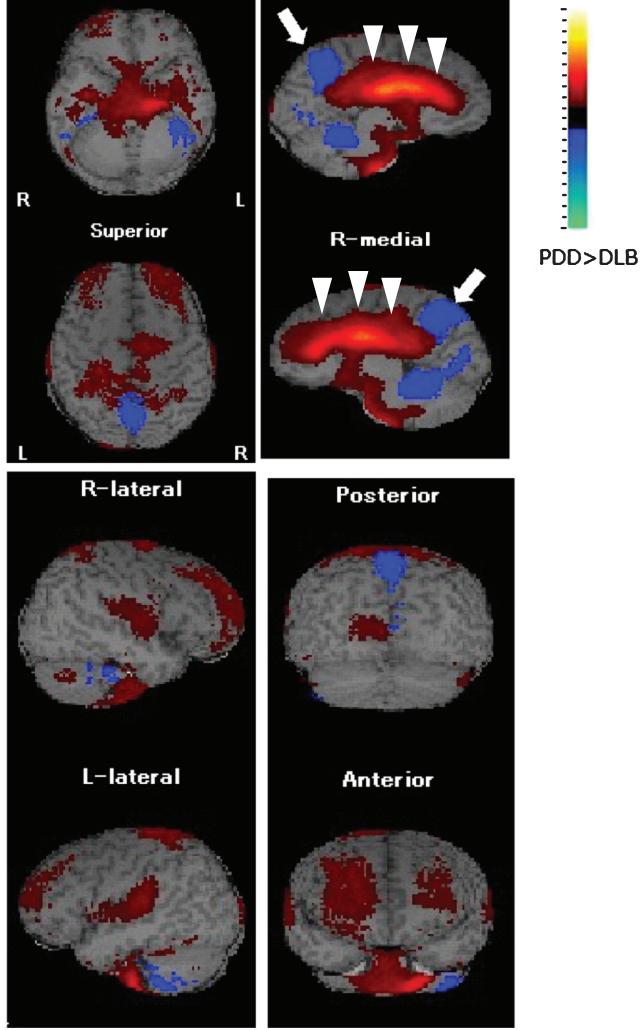

Fig. 1. ${ }^{99 m}$ Tc-ECD SPECT images of the mean values in PDD and DLB patients (left panels). There is a slight decrease of CBF in the frontal lobe in PDD, but similar CBF levels in the posterior lobes of both PDD and DLB. Subtraction image of mean PDD - mean DLB (right panels), showing a higher CBF in whole cingulate gyrus in DLB than PDD groups (arrowheads), and a lower CBF in the precuneus area in DLB (arrow) than PDD.

difference between PDD and DLB. Because the ABS revealed high scores for hallucination, delusion, wandering and abnormal behavior, it could be a useful measure for distinguishing DLB from PDD.

Autonomic dysfunction is common in both PDD and DLB, but $\mathrm{OH}$ was much more common in DLB (78.0\%, Table 1) than PDD (7.0\%). Previous reports have found autonomic dysfunction [20] in $95 \%$ of DLB and $60 \%$ of PD patients, and $\mathrm{OH}$ in $62.5 \%$ of PDD patients [21]. This high rate of $\mathrm{OH}$ could also be a useful indicator for DLB over PDD.

ADL scores were significantly worse in PDD than DLB (Table 2), probably owing to the higher rates of tremor $(p<0.001)$, rigidity, postural instability $(p=0.022)$, urinary disturbance, and constipation in PDD (Table 1). A previous report showed that $83 \%$ of PDD (age 73.1 \pm 5.8 ) and $73 \%$ of DLB patients (age $76.4 \pm 6.8$ ) suffered from these symptoms [22]. These data may correspond to the poorer Yahr scores (mean $2.8 \pm 0.8$, Yahr 4 is 18.4\%) in PDD than DLB (mean $2.4 \pm 0.8$, Yahr 4 is $5.4 \%$ ). Our study shows similar abnormalities of white matter MRI and autonomic MIBG, suggesting a faster progression of such structural and functional abnormalities in DLB than in PDD (Table 2), even though the DLB patients had a shorter disease duration and younger age than the PDD group (Table 1).

Subtraction image of averaged PDD and DLB SPECT image analysis (DLB $>$ PDD region is shown as the red to yellow colored region, whereas the PDD $>$ DLB region is shown as blue to green) showed, for the first time, the interesting result that PDD patients have lower CBF in the whole cingulate gyrus area and that DLB patients have lower $\mathrm{CBF}$ in the precuneus area (Figure, right). Both areas involved in Alzheimer's disease. Previous studies showed decreases of CBF in the posterior cingulate cortex, precuneus, and bilateral parietotemporal 
association cortex in PDD [9], and in the occipital lobe, percutaneous gyrus and posterior cingulate cortex in DLB [7]. From these result, we speculate that PDD patients' relatively poor average subscale scores for "repetition" (MMSE), "recent memory" (HDS$\mathrm{R})$, and "lexical fluency" (FAB) could be due to lower CBF in the cingulate gyrus than in DLB. In addition, DLB patients' poorer average subscale scores of "orientation to place" (MMSE), "similarities", "conflicting instructions", and "go-no go" (FAB) could be due to the lower CBF in the precuneus area in DLB than PDD. In addition, previous study demonstrated that cerebral perfusion patterns in $\mathrm{AD}$ and PDD are similar [23], making it difficult to describe the CBF pattern of PDD patients. We suspect this difficulty is one of the reasons that there are no previous reports comparing regional hypoperfusion between PDD and DLB.

In conclusion, the present study demonstrates for the first time that patients with PDD show significantly better FAB and "orientation to place" scores than DLB patients as assessed by the MMSE and HDS-R. In addition, PDD patients also have significantly better GDS and ABS scores. PDD-DLB subtraction SPECT images demonstrated differences between PDD and DLB.

\section{ACKNOWLEDGMENTS}

This work was supported in part by Grants-inAid for Scientific Research (B) 2529320216 and (C) 24591263, and Challenging Research grant 24659651. Mizusawa H, Nakano I, Nishizawa M, Sasaki H, and Aoki M received Grants-in-Aid from the Research Committees of the Ministry of Health, Labour and Welfare of Japan.

Authors' disclosures available online (http://jalz.com/manuscript-disclosures/15-0952r2).

\section{REFERENCES}

[1] Lang AE, Obeso JA (2004) Challenges in Parkinson's disease: Restoration of the nigrostriatal dopamine system is not enough. Lancet Neurol 3, 309-316.

[2] Hely MA, Reid WG, Adena MA, Halliday GM, Morris JG (2008) The Sydney multicenter study of Parkinson's disease: The inevitability of dementia at 20 years. Mov Disord 23, 837-844.

[3] Lim SY, Lang AE (2010) The nonmotor symptoms of Parkinson's disease-an overview. Mov Disord 25(Suppl 1), S123-S130.

[4] Buter TC, van den Hout A, Matthews FE, Larsen JP, Brayne C, Aarsland D (2008) Dementia and survival in Parkin- son disease: A 12-year population study. Neurology 70, 1017-1022.

[5] Hishikawa N, Fukui Y, Sato K, Kono S, Yamashita T, Ohta Y, Deguchi K, Abe K (2015) Characteristic features of cognitive, affective and daily living functions of late-elderly dementia. Geriatr Gerontol Int, doi: 10.1111/ ggi. 12492

[6] Park KW, Kim HS, Cheon SM, Cha JK, Kim SH, Kim JW (2011) Dementia with Lewy bodies versus Alzheimer's disease and Parkinson's disease dementia: A comparison of cognitive profiles. J Clin Neurol 7, 19-24.

[7] Waragai M, Yamada T, Matsuda H (2007) Evaluation of brain perfusion SPECT using an easy Z-score imaging system (eZIS) as an adjunct to early-diagnosis of neurodegenerative diseases. J Neurol Sci 260, 57-64.

[8] Shimizu S, Hanyu H, Kanetaka H, Iwamoto T, Koizumi K, Abe K (2005) Differentiation of dementia with Lewy bodies from Alzheimer's disease using brain SPECT. Dement Geriatr Cogn Disord 20, 25-30.

[9] Imamura K, Wada-Isoe K, Kowa H, Tanabe Y, Nakashima $\mathrm{K}$ (2008) The effect of donepezil on increased regional cerebral blood flow in the posterior cingulate cortex of a patient with Parkinson's disease dementia. Neurocase 14, 271-275.

[10] Dubois B, Tolosa E, Katzenschlager R, Emre M, Lees AJ, Schumann G, Pourcher E, Gray J, Thomas G, Swartz J, Hsu T, Moline ML (2012) Donepezil in Parkinson's disease dementia: A randomized, double-blind efficacy and safety study. Mov Disord 27, 1230-1238.

[11] Mori E, Ikeda M, Kosaka K (2012) Donepezil for dementia with Lewy bodies: A randomized, placebo-controlled trial. Ann Neurol 72, 41-52.

[12] Emre M, Cummings JL, Lane RM (2007) Rivastigmine in dementia associated with Parkinson's disease and Alzheimer's disease: Similarities and differences. J Alzheimers Dis 11, 509-519.

[13] McKeith IG, Dickson DW, Lowe J, Emre M, O'Brien JT, Feldman H, Cummings J, Duda JE, Lippa C, Perry EK, Aarsland D, Arai H, Ballard CG, Boeve B, Burn DJ, Costa D, Del Ser T, Dubois B, Galasko D, Gauthier S, Goetz CG, Gomez-Tortosa E, Halliday G, Hansen LA, Hardy J, Iwatsubo T, Kalaria RN, Kaufer D, Kenny RA, Korczyn A, Kosaka K, Lee VM, Lees A, Litvan I, Londos E, Lopez OL, Minoshima S, Mizuno Y, Molina JA, Mukaetova-Ladinska EB, Pasquier F, Perry RH, Schulz JB, Trojanowski JQ, Yamada M (2005) Diagnosis and management of dementia with Lewy bodies: Third report of the DLB Consortium. Neurology 65, 1863-1872.

[14] Fazekas F, Chawluk JB, Alavi A, Hurtig HI, Zimmerman RA (1987) MR signal abnormalities at 1.5 T in Alzheimer's dementia and normal aging. AJR Am J Roentgenol 149, 351-356.

[15] Matsuda H, Mizumura S, Nagao T, Ota T, Iizuka T, Nemoto K, Takemura N, Arai H, Homma A (2007) Automated discrimination between very early Alzheimer disease and controls using an easy Z-score imaging system for multicenter brain perfusion single-photon emission tomography. AJNR Am J Neuroradiol 28, 731-736.

[16] Nakatsuka M, Meguro K, Tsuboi H, Nakamura K, Akanuma K, Yamaguchi S (2013) Content of delusional thoughts in Alzheimer's disease and assessment of content-specific brain dysfunctions with BEHAVE-AD-FW and SPECT. Int Psychogeriatr 25, 939-948.

[17] Yamane Y, Sakai K, Maeda K (2011) Dementia with Lewy bodies is associated with higher scores on the Geriatric 
Depression Scale than is Alzheimer's disease. Psychogeriatrics 11, 157-165.

[18] Caballol N, Marti MJ, Tolosa E (2007) Cognitive dysfunction and dementia in Parkinson disease. Mov Disord 22(Suppl 17), S358-S366.

[19] Abe K, Yamashita T, Hishikawa N, Ohta Y, Deguchi K, Sato K, Matsuzono K, Nakano Y, Ikeda Y, Wakutani Y, Takao Y (2015) A new simple score (ABS) for assessing behavioral and psychological symptoms of dementia. J Neurol Sci 350, 14-17.

[20] Thaisetthawatkul P, Boeve BF, Benarroch EE, Sandroni P, Ferman TJ, Petersen R, Low PA (2004) Autonomic dysfunction in dementia with Lewy bodies. Neurology 62, 1804-1809.
[21] Peralta C, Stampfer-Kountchev M, Karner E, Kollensperger M, Geser F, Wolf E, Seppi K, Benke T, Poewe W, Wenning GK (2007) Orthostatic hypotension and attention in Parkinson's disease with and without dementia. J Neural Transm 114, 585-588.

[22] Burn DJ (2005) Update on dementia with Lewy bodies. Curr Neurol Neurosci Rep 5, 339-344.

[23] Le Heron CJ, Wright SL, Melzer TR, Myall DJ, MacAskill MR, Livingston L, Keenan RJ, Watts R, Dalrymple-Alford JC, Anderson TJ (2014) Comparing cerebral perfusion in Alzheimer's disease and Parkinson's disease dementia: An ASL-MRI study. J Cereb Blood Flow Metab 34, 964-970. 